Check for updates

Cite this: RSC Adv., 2018, 8, 31296

Received 20th June 2018

Accepted 23rd August 2018

DOI: $10.1039 / \mathrm{c} 8 \mathrm{ra05270k}$

rsc.li/rsc-advances

\section{Flexible solid-state supercapacitor based on tin oxide/reduced graphene oxide/bacterial nanocellulose $\uparrow$}

\author{
Keng-Ku Liu, ${ }^{a}$ Qisheng Jiang, ${ }^{a}$ Clayton Kacica, ${ }^{b}$ Hamed Gholami Derami, ${ }^{a}$ \\ Pratim Biswas (D) ${ }^{* b}$ and Srikanth Singamaneni (D)*a
}

\begin{abstract}
We demonstrate a flexible and light-weight supercapacitor based on bacterial nanocellulose (BNC) incorporated with tin oxide $\left(\mathrm{SnO}_{2}\right)$ nanoparticles, graphene oxide (GO) and poly(3,4ethylenedioxyiophene)-poly(styrenesulfonate) (PEDOT:PSS). The $\mathrm{SnO}_{2}$ and $\mathrm{GO}$ flakes are introduced into the fibrous nanocellulose matrix during bacteria-mediated synthesis. The flexible PEDOT:PSS/SnO $/ 2 / \mathrm{rGO} /$ BNC electrodes exhibited excellent electrochemical performance with a capacitance of $445 \mathrm{~F} \mathrm{~g}^{-1}$ at $2 \mathrm{~A} \mathrm{~g}^{-1}$ and outstanding cycling stability with $84.1 \%$ capacitance retention over 2500 charge/discharge cycles. The flexible solid-state supercapacitors fabricated using PEDOT:PSS $/ \mathrm{SnO}_{2} / \mathrm{rGO} / \mathrm{BNC}$ electrodes and poly(vinyl alcohol) (PVA)- $\mathrm{H}_{2} \mathrm{SO}_{4}$ coated $\mathrm{BNC}$ as a separator exhibited excellent energy storage performance. The fabrication method demonstrated here is highly scalable and opens up new opportunities for the fabrication of flexible cellulose-based energy storage devices.
\end{abstract}

\section{Introduction}

Flexible energy storage devices are extremely important for a wide range of flexible and wearable electronic and optoelectronic devices that are finding numerous applications in healthcare and consumer electronics. ${ }^{1-4}$ Supercapacitors have attracted extensive attention as flexible energy storage devices due to their higher power density, rapid charge-discharge characteristics, and long cycle life. ${ }^{5,6}$ Owing to their high surface area, high electrical conductivity and superior stability, carbonbased materials such as carbon nanotubes (CNT), carbon nanofibers (CNF), graphene, graphene oxide (GO) and activated carbon are widely employed in electric double-layer capacitors (EDLCs). $)^{3,5,7-13}$ Pseudocapacitive materials (e.g. conducting polymers and metal oxides) are commonly integrated with EDLCs to form hybrid supercapacitors with enhanced capacitance and energy densities owing to the fast and highlyreversible faradic processes between the electroactive species on the surface of the electrode and the electrolyte. ${ }^{6,14-19}$ Among the metal oxides, tin oxide $\left(\mathrm{SnO}_{2}\right)$ has attracted much attention due to its high theoretical capacity $\left(\sim 782 \mathrm{~mA} \mathrm{~h} \mathrm{~g}^{-1}\right)$, high abundance and low cost. ${ }^{20-23}$

\footnotetext{
${ }^{a}$ Department of Mechanical Engineering and Materials Science, Institute of Materials Science and Engineering, Washington University in St. Louis, St Louis, Missouri 63130,USA.E-mail: singamaneni@wustl.edu

${ }^{b}$ Department of Energy, Environmental and Chemical Engineering, Washington University in St. Louis, St Louis, Missouri 63130, USA. E-mail: pbiswas@wustl.edu

$\dagger$ Electronic supplementary information (ESI) available. See DOI: $10.1039 / \mathrm{c} 8 \mathrm{ra} 05270 \mathrm{k}$
}

Cellulosic materials are considered to be highly promising for flexible electrical energy storage devices due to their flexibility, highly porous structure, lightweight, and low cost. ${ }^{14,24-30}$ It has been demonstrated that a composite structure comprised of cellulose paper and carbon materials exhibited promising energy-storage performance. ${ }^{7,8,25,30}$ Recently, Ko and co-workers have reported flexible supercapacitor electrodes composed of metal and pseudocapacitive nanoparticles on a metal-like paper, which significantly increases the areal capacitance and rate capability. ${ }^{24}$ Chen et al. have demonstrated an all-woodstructured supercapacitor, which exhibited high energy storage capacity. ${ }^{31}$ The components of this all-wood-structured supercapacitor are biocompatible, environmentally friendly, and low-cost. ${ }^{31}$ More recently, we have demonstrated an in situ formation of $\mathrm{GO} /$ bacterial nanocellulose (BNC) composite as light-weight supercapacitor electrode. ${ }^{32}$ Additionally, this GO/ BNC-based supercapacitor exhibited excellent energy storage properties and stability. ${ }^{32}$ These recent reports further signify the advantages of cellulose for the applications in energy storage. However, compared to hybrid supercapacitors, EDLCs exhibit relative low specific capacitance. Thus, the combination of cellulosic materials-based EDLCs with pseudocapacitive materials to form hybrid supercapacitors is considered to be highly promising for flexible energy storage devices with enhanced energy storage performance.

In this work, we demonstrate the incorporation of $\mathrm{SnO}_{2}$ and GO flakes into nanofibrous cellulose matrix during its bacteriamediated growth followed by coating with a conductive polymer, poly(3,4-ethylenedioxyiophene)-poly(styrenesulfonate) (PEDOT:PSS), to realize a flexible, light-weight, and solid-state 
supercapacitor. The flexible electrodes exhibit excellent electrochemical performance with capacitance of $445 \mathrm{~F} \mathrm{~g}^{-1}$ at $2 \mathrm{~A} \mathrm{~g}^{-1}$ and the long-term stability (retained $84.1 \%$ capacitance after 2500 charge/discharge cycles). The flexible solid-state supercapacitor fabricated using PEDOT:PSS $/ \mathrm{SnO}_{2} / \mathrm{rGO} / \mathrm{BNC}$ as electrodes and poly(vinyl alcohol) (PVA) $-\mathrm{H}_{2} \mathrm{SO}_{4}$ coated $\mathrm{BNC}$ as separator showed excellent energy storage performance. Furthermore, the integrated device showed small capacitance decay over 2500 charge/discharge cycles.

\section{Experimental section}

\subsection{Materials}

Potassium stannate trihydrate (99.9\% trace metals basis), urea, graphite flakes, potassium permanganate, hydrogen peroxide, hydrochloric acid, PEDOT:PSS $\left(1.0 \mathrm{wt} \%\right.$ in $\mathrm{H}_{2} \mathrm{O}$, highconductivity grade), hypophosphorous acid (HPA, 50\%), iodine ( $\mathrm{I}_{2}$ ), and poly(vinyl alcohol) (PVA, $M_{\mathrm{w}}$ 146000-186000, 99+\% hydrolyzed) were purchased from Sigma-Aldrich.

\subsection{Preparation of $\mathrm{SnO}_{2}$ nanoparticles}

$0.12 \mathrm{~g}$ of urea was added into $20 \mathrm{ml}$ mixture containing ethanol and water with a volume ratio of $3: 5.0 .096 \mathrm{~g}$ of potassium stannate trihydrate was slowly added into this mixture with constant stirring. The mixture solution was transferred to a Teflon-lined stainless steel autoclave and heated in an oven at $190{ }^{\circ} \mathrm{C}$ for $24 \mathrm{~h}$. The product was centrifuged and washed with nanopure water and ethanol several times and redispersed in nanopure water.

\subsection{Preparation of PEDOT:PSS/ $\mathrm{SnO}_{2} / \mathrm{rGO} / \mathrm{BNC}$ electrodes}

GO was prepared using the procedure reported by Marcano et $a l .^{33}$ Gluconacetobacter hansenii (ATCC ${ }^{3} 53582$ ) was cultured in test tubes containing $16 \mathrm{ml}$ of $\# 1765$ medium at $30{ }^{\circ} \mathrm{C}$ under shaking at $250 \mathrm{rpm}$. The \#1765 medium is composed of $2 \%(\mathrm{w} /$ v) glucose, $0.5 \%(\mathrm{w} / \mathrm{v})$ yeast extract, $0.5 \%(\mathrm{w} / \mathrm{v})$ peptone, $0.27 \%$ $(\mathrm{w} / \mathrm{v})$ disodium phosphate, and $0.5 \%(\mathrm{w} / \mathrm{v})$ citric acid. Graphene oxide solution ( $5 \mathrm{ml}$ of $0.3 \mathrm{wt} \%$ ) was centrifuged and redispersed in $\$ 1765$ medium and then centrifuged again to leave a wet mixture of GO and medium after decanting supernatant. $\mathrm{SnO}_{2}$ nanoparticles were washed with $\# 1765$ medium, centrifuged and then re-dispersed in $\# 1765$ medium. Bacterial culture solution (incubated 3 days) and $\mathrm{SnO}_{2}$ nanoparticles in $\# 1765$ medium were added to the $\mathrm{GO} /$ medium wet mixture to make it to a total of $15 \mathrm{ml}$ (with GO concentration of $0.1 \mathrm{wt} \%$, $\mathrm{SnO}_{2}$ concentration of $0.5 \mathrm{mg} \mathrm{ml}^{-1}$ ) and mixed thoroughly. The solution was transferred to a Petridish and incubated at room temperature for 7 days. The BNC film was harvested and washed in $0.1 \mathrm{M} \mathrm{NaOH}$ aqueous solution under boiling condition for $2 \mathrm{~h}$ then washed in nanopure water. The reduction of graphene oxide was performed according to the procedure reported by Pham et al..$^{34}$ The washed $\mathrm{SnO}_{2} / \mathrm{GO} / \mathrm{BNC}$ hydrogel was immersed in a $300 \mathrm{ml}$ solution containing $100 \mathrm{~g}$ of HPA and $5 \mathrm{~g}$ of $\mathrm{I}_{2}$ and heated at $85{ }^{\circ} \mathrm{C}$ for $12 \mathrm{~h}$. Then the $\mathrm{SnO}_{2} / \mathrm{rGO} / \mathrm{BNC}$ was washed with nanopure water and dried. PEDOT:PSS was added on the $\mathrm{SnO}_{2} / \mathrm{rGO} / \mathrm{BNC}$ film and dried under the ambient condition to form PEDOT:PSS/SnO $/ 2 / \mathrm{rGO} / \mathrm{BNC}$ electrodes. The mass of the electrodes was measured using microbalance, and the mass of active materials loading is measured to be $0.57 \mathrm{mg} \mathrm{cm}^{-2}$.

\subsection{Preparation of flexible solid-state supercapacitor}

Electrolyte was prepared by mixing $2 \mathrm{~g}$ of PVA and $2 \mathrm{~g}$ of $\mathrm{H}_{2} \mathrm{SO}_{4}$ in $20 \mathrm{ml}$ of nanopure water and then heated at $85{ }^{\circ} \mathrm{C}$ until the solution became clear. After the $\mathrm{PVA}-\mathrm{H}_{2} \mathrm{SO}_{4}$ electrolyte was cooled to room temperature, a piece of BNC film was immersed in it for $10 \mathrm{~min}$. Then the PVA- $\mathrm{H}_{2} \mathrm{SO}_{4}$ coated BNC film was sandwiched between PEDOT:PSS $/ \mathrm{SnO}_{2} / \mathrm{rGO} / \mathrm{BNC}$ electrodes and dried at room temperature overnight to form a flexible solid-state supercapacitor.

\subsection{Characterization}

SEM images were collected using FEI Nova NanoSEM 2300. AFM image was collected from Dimension 3000 (Bruker). Raman spectrum was collected from Renishaw inVia confocal Raman microscope. XPS spectrum was collected from VersaProbe II Scanning ESCA Microprobe. Electrical conductivity measurement is perform using the four-point probe station. ${ }^{35}$

\subsection{Electrochemical measurement}

All electrochemical measurements were performed using a CHI 760 bipotentiostat ( $\mathrm{CH}$ Instruments, Austin, USA). Cyclic (a)

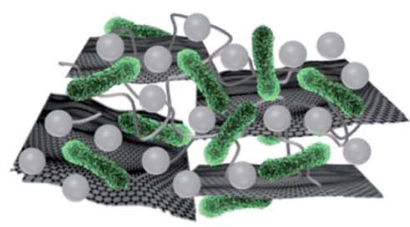

Gluconacetobacter hansenii
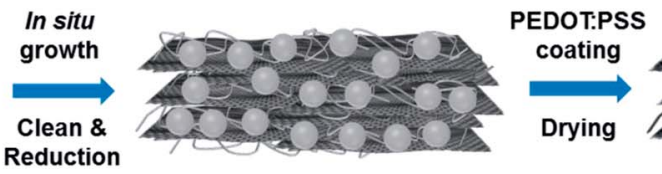

Graphene oxide (b)

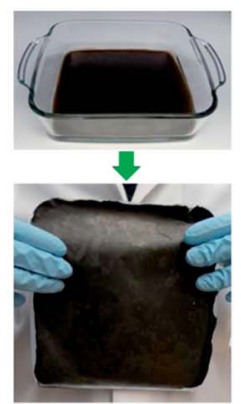

Fig. 1 (a) Schematic illustration showing the synthesis process of the PEDOT:PSS/SnO$/ 2 / r G O / B N C$ electrode for supercapacitor. (b) Photographs of the $\mathrm{SnO}_{2} / \mathrm{GO} / \mathrm{BNC}$ in hydrogel and dried states. 
voltammetry was performed over a potential range of 0 to $1 \mathrm{~V}$ at scan rates of $5,10,25,50,100,150$, and $250 \mathrm{mV} \mathrm{s}^{-1}$. Galvanostatic charge/discharge studies were carried out using a chronopotentiometry technique over a potential range of 0 to $1 \mathrm{~V}$. Electrochemical studies were carried out using a three-electrode configuration for the single electrode and using a two-electrode configuration for the solid-state device. The three electrode configuration utilized the PEDOT:PSS $/ \mathrm{SnO}_{2} / \mathrm{rGO} / \mathrm{BNC}$ composite as the working electrode, a platinum wire counter electrode, and an $\mathrm{Ag} / \mathrm{AgCl}$ reference electrode, in a $1 \mathrm{M} \mathrm{H}_{2} \mathrm{SO}_{4}$ electrolyte solution.

\section{Results and discussion}

The fabrication of PEDOT:PSS $/ \mathrm{SnO}_{2} / \mathrm{rGO} / \mathrm{BNC}$ electrode involves the culture of nanocellulose producing bacteria, Gluconacetobacter hansenii, in the presence of $\mathrm{SnO}_{2}$ nanoparticles and GO flakes, followed by the reduction of GO and PEDOT:PSS coating (Fig. 1a). GO flakes were prepared according to a procedure reported previously with slight modification. ${ }^{33}$ Atomic Force Microscope (AFM) image revealed the thickness of GO sheets to be around $1 \mathrm{~nm}$ (Fig. S1a in ESI $\dagger$ ). Two characteristic bands at $\sim 1591$ and $\sim 1352 \mathrm{~cm}^{-1}$ were observed in the
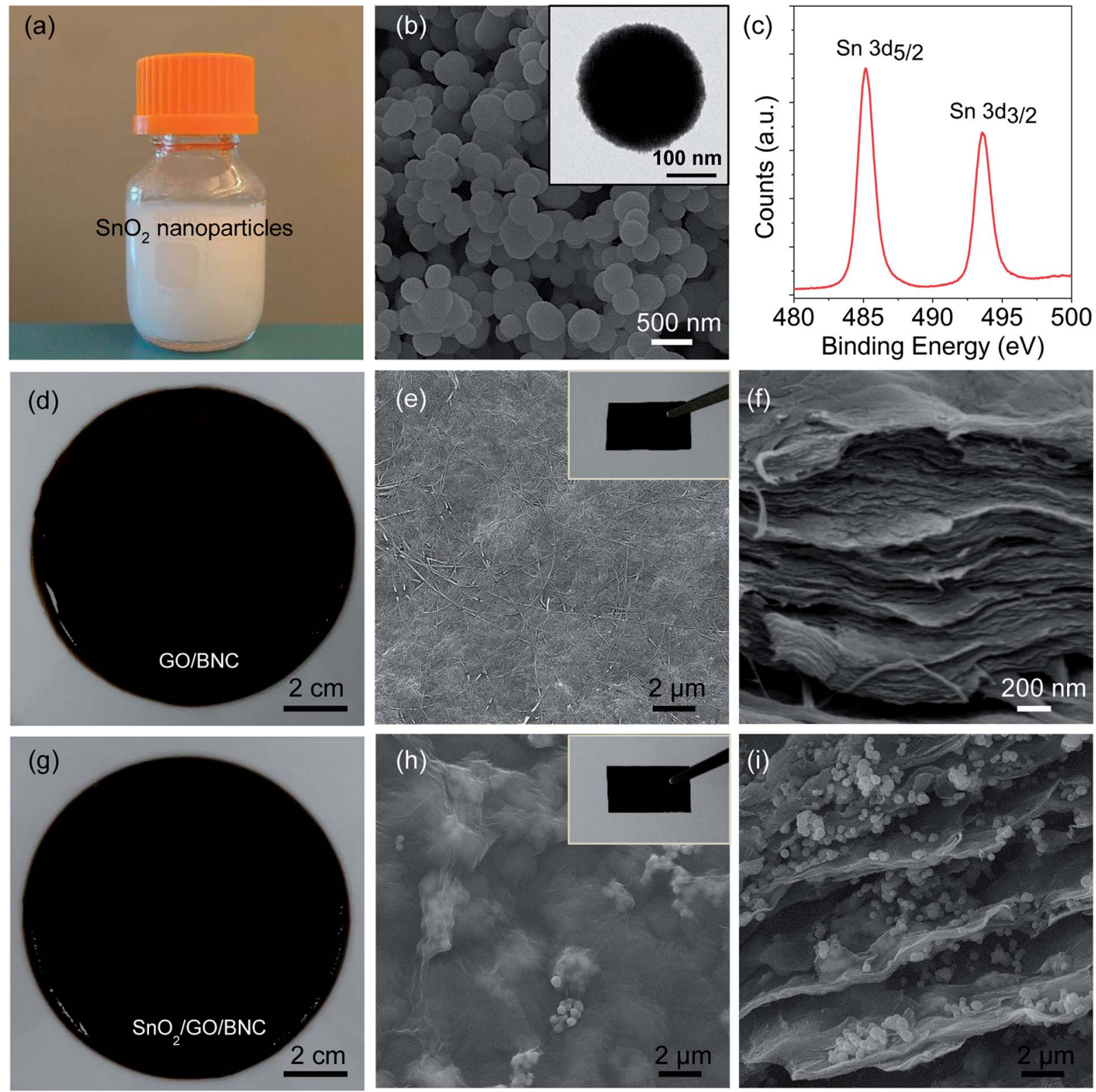

Fig. 2 (a) Photograph of the $\mathrm{SnO}_{2}$ nanoparticles aqueous solution. (b) SEM image of the $\mathrm{SnO}_{2}$ nanoparticles. Inset shows the TEM image of the $\mathrm{SnO}_{2}$ nanoparticle. (c) X-ray photoelectron spectrum of the $\mathrm{SnO}_{2}$ nanoparticles. (d) Photograph of the GO/BNC hydrogel. (e) SEM image of the top surface of the GO/BNC. Inset shows the photograph of the GO/BNC. (f) Cross-sectional SEM image of the GO/BNC. (g) Photograph of the $\mathrm{SnO}_{2} / \mathrm{GO} / \mathrm{BNC}$ hydrogel. (h) SEM image of the top surface of the $\mathrm{SnO}_{2} / \mathrm{GO} / \mathrm{BNC}$. Inset shows the digital image of the $\mathrm{SnO} / \mathrm{GO} / \mathrm{BNC}$ film. (i) Cross-sectional SEM image of the $\mathrm{SnO}_{2} / \mathrm{GO} / \mathrm{BNC}$. 
Raman spectrum of GO, which correspond to the G-band and Dband, respectively (Fig. S1b in $\mathrm{ESI}^{\dagger}$ ). $\mathrm{SnO}_{2}$ nanoparticles were synthesized according to a procedure reported by Zhang et al.

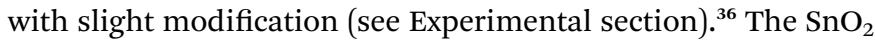
growth solution is comprised of urea, potassium stannate trihydrate, and a mixture of ethanol and water. The mixture solution was placed in an autoclave reactor and heated in an oven at $190{ }^{\circ} \mathrm{C}$ for 24 hours. The aqueous solution of $\mathrm{SnO}_{2}$ nanoparticles was found to be milky (Fig. 2a). Scanning and transmission electron microscope (SEM and TEM) images revealed the size of the $\mathrm{SnO}_{2}$ nanoparticles to be 250-300 nm (Fig. 2b). X-ray photoelectron spectroscopy (XPS) was employed to investigate the surface chemical composition of the $\mathrm{SnO}_{2}$ nanoparticles (Fig. 2c). Two strong peaks at around $485 \mathrm{eV}$ and $494 \mathrm{eV}$ can be attributed to $\mathrm{Sn} 3 \mathrm{~d}_{5 / 2}$ and $\mathrm{Sn} 3 \mathrm{~d}_{3 / 2}$, respectively, which confirm the formation of $\mathrm{SnO}_{2}$ nanoparticles. ${ }^{21,37,38}$ In a typical experiment for the preparation of $\mathrm{SnO}_{2} / \mathrm{GO} / \mathrm{BNC}$ film, GO flakes and $\mathrm{SnO}_{2}$ nanoparticles were washed and redispersed in $\# 1765$ medium to form $\mathrm{GO} /$ medium and $\mathrm{SnO}_{2} /$ medium, separately (see Experimental section for details). A mixture solution containing a predetermined concentration of GO flakes and $\mathrm{SnO}_{2}$ in the medium was homogeneously mixed with bacteria and set aside under static condition at room temperature for seven days for the formation of $\mathrm{SnO}_{2} / \mathrm{GO} / \mathrm{BNC}$ film in a semi-dry state (Fig. 1b top). The obtained $\mathrm{SnO}_{2} / \mathrm{GO} /$ BNC film was washed in boiling $\mathrm{NaOH}$ solution and nanopure water. To reduce the GO, the washed $\mathrm{SnO}_{2} / \mathrm{GO} / \mathrm{BNC}$ film was immersed in a mixture of hypophosphorous acid (HPA) and iodine $\left(\mathrm{I}_{2}\right)$ according to previous report. ${ }^{34}$ Then the $\mathrm{SnO}_{2} / \mathrm{rGO} /$ BNC was washed with nanopure water and dried (Fig. 1b bottom). Fig. $2 \mathrm{~d}$ and $\mathrm{g}$ show the semi-dry GO/BNC film without and with $\mathrm{SnO}_{2}$ loading, respectively. SEM image of the GO/BNC film revealed an entangled network of BNC nanofibers and GO flakes embedded within the fiber network (Fig. 2e). The crosssectional SEM image revealed the layered structure of GO/BNC film (Fig. 2f). SEM images of the $\mathrm{SnO}_{2} / \mathrm{GO} / \mathrm{BNC}$ film revealed the $\mathrm{SnO}_{2}$ nanoparticles embedded within the $\mathrm{GO} / \mathrm{BNC}$ layers (Fig. $2 \mathrm{~h}$ and i). The electrical conductivity of $\mathrm{SnO}_{2} / \mathrm{rGO} / \mathrm{BNC}$ electrode exhibited a slight decrease with increasing the $\mathrm{SnO}_{2}$ concentrations (Fig S2 $\dagger$ ) owing to the low conductivity nature of $\mathrm{SnO}_{2}$. The conducting polymer, PEDOT:PSS, was added onto the $\mathrm{SnO}_{2} / \mathrm{rGO} / \mathrm{BNC}$ film, uniformly spread and dried under the ambient condition to form PEDOT:PSS $/ \mathrm{SnO}_{2} / \mathrm{rGO} / \mathrm{BNC}$ electrodes.

Next, the electrochemical performance of the electrodes was investigated in a three-electrode configuration in $1 \mathrm{M} \mathrm{H}_{2} \mathrm{SO}_{4}$ electrolyte using cyclic voltammetry (CV) and galvanostatic charge-discharge (GCD) techniques. Presence of $\mathrm{SnO}_{2}$ nanoparticles in the electrode results in a higher specific capacitance as evidenced by the CV curves. The specific capacitance (as represented by the integrated area in the $\mathrm{CV}$ curve) of PEDOT:PSS $/ \mathrm{SnO}_{2} / \mathrm{rGO} / \mathrm{BNC}\left(402 \mathrm{~F} \mathrm{~g}^{-1}\right)$ is significantly higher than that of PEDOT:PSS/rGO/BNC $\left(123 \mathrm{~F} \mathrm{~g}^{-1}\right)$ due to highly reversible faradaic redox processes associated with to $\mathrm{SnO}_{2}$ (Fig. 3a). The fraction of pseudocapacitance in the total capacitance is calculated to be around $69 \%$. The peak current of

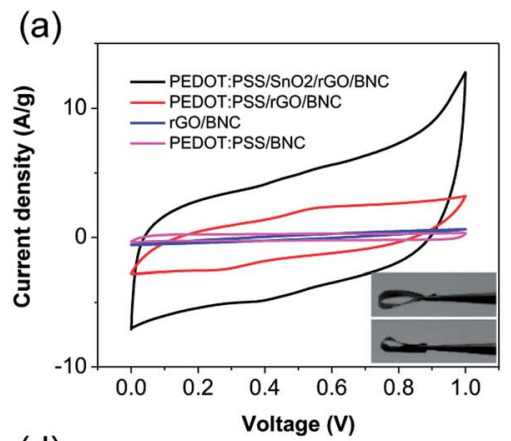

(d)

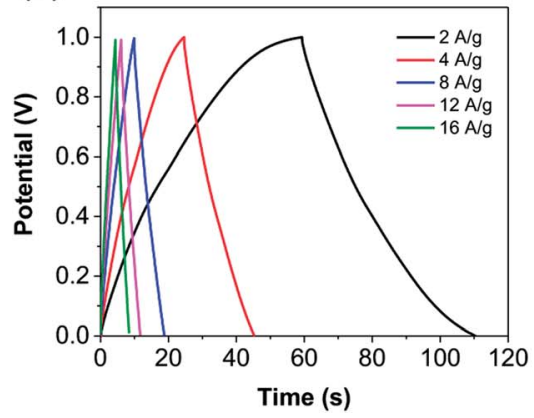

(b)

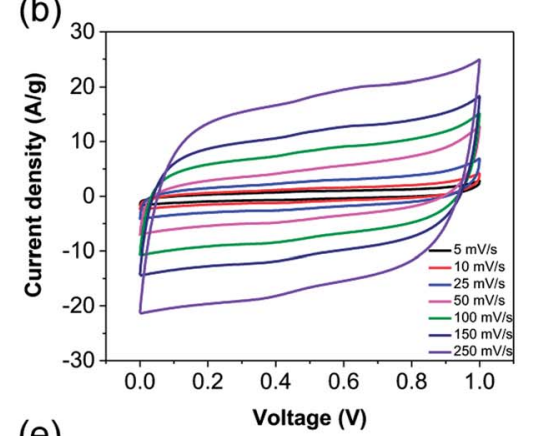

(e)

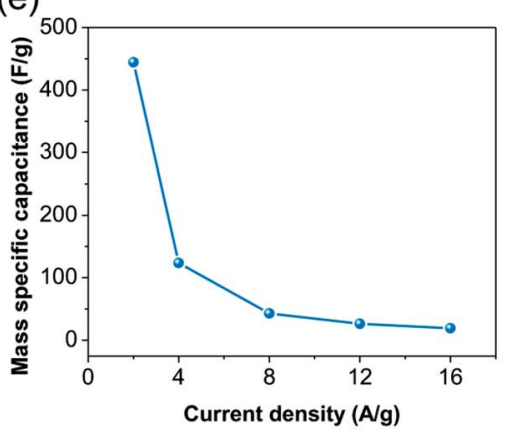

(c)

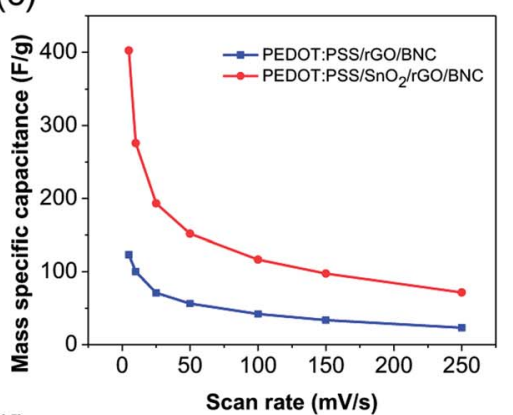

(f)

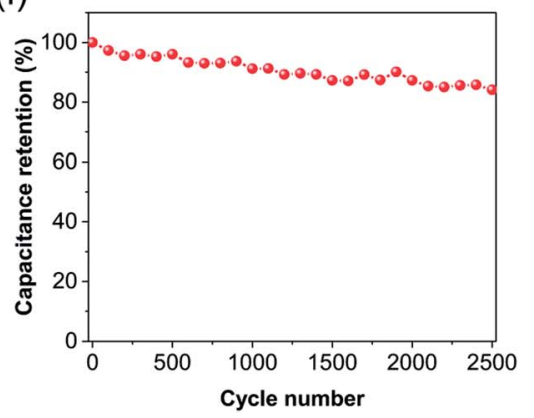

Fig. 3 Electrochemical performance of the PEDOT:PSS/rGO/BNC electrodes. (a) CV curves of the rGO/BNC, PEDOT:PSS/BNC, PEDOT:PSS/ $\mathrm{rGO} / \mathrm{BNC}$ and PEDOT:PSS/SnO $/ 2 / \mathrm{rGO} / \mathrm{BNC}$ electrodes at the scan rate of $50 \mathrm{mV} \mathrm{s}^{-1}$. Insets show the digital images of the PEDOT:PSS/rGO/BNC electrodes without (top) and with (down) $\mathrm{SnO}_{2}$ nanoparticles. (b) CV curves collected from various scan rates. (c) Mass specific capacitance with (red) and without (blue) $\mathrm{SnO}_{2}$ nanoparticles at various scan rates. (d) Charge-discharge curves at various current densities. (e) Mass specific capacitance of the electrode with $\mathrm{SnO}_{2}$ calculated from the charge-discharge curves at various current densities. (f) Cycling performance of the PEDOT:PSS/SnO $2 / \mathrm{rGO} / \mathrm{BNC}$ electrode. 
PEDOT:PSS $/ \mathrm{SnO}_{2} / \mathrm{rGO} / \mathrm{BNC}$ electrodes exhibited an increase in the peak current upon scanning from 5 to $250 \mathrm{mV} \mathrm{s}^{-1}$ (Fig. 3b). The CV curves largely retain their rectangular shape, representative of an ideal capacitor, suggesting a fast charge transfer rate even at high scan rates. Specific capacitance $\left(C_{\mathrm{s}, \mathrm{CV}}\right)$ of the electrodes decreased from $402 \mathrm{~F} \mathrm{~g}^{-1}$ to $72 \mathrm{~F} \mathrm{~g}^{-1}$ as the scan rate was increased from 5 to $250 \mathrm{mV} \mathrm{s}^{-1}$ (Fig. 3c). At the same scan rate, PEDOT:PSS/SnO ${ }_{2} / \mathrm{rGO} / \mathrm{BNC}$ electrodes exhibited higher specific capacitance compared to PEDOT:PSS/rGO/BNC. GCD curves collected at different current densities (2 to $16 \mathrm{~A} \mathrm{~g}^{-1}$ ) exhibited typical triangular shape with symmetric chargedischarge profiles, further confirming the fast charge transfer rate (Fig. 3d). Additionally, only a small ohmic (IR) drop was observed at a scan rate of $16 \mathrm{~A} \mathrm{~g}^{-1}$. The specific capacitance $\left(C_{\mathrm{S}, \mathrm{GCD}}\right)$ of electrodes was found to decrease from $445 \mathrm{~F} \mathrm{~g}^{-1}$ to 19 $\mathrm{F} \mathrm{g}^{-1}$ as the current density was increased from 2 to $16 \mathrm{~A} \mathrm{~g}^{-1}$ (Fig. 3e). The longevity and stability of the PEDOT:PSS/SnO $\mathrm{S}_{2} /$ rGO/BNC electrodes was evaluated using GCD technique for 2500 cycles at a current density of $2 \mathrm{~A} \mathrm{~g}^{-1}$ (Fig. 3f). The electrode retains $84.1 \%$ of its initial capacitance over 2500 chargedischarge cycles, indicating this composite electrode is a promising candidate for supercapacitor devices.

The solid-state supercapacitor devices were prepared using two electrodes separated by a BNC film saturated with PVA$\mathrm{H}_{2} \mathrm{SO}_{4}$ as electrolyte (Fig. 4a). Considering that the electrode fabrication approach is highly scalable, large electrodes and separator films can be easily synthesized and assembled into a flexible device (Fig. 4b right and middle respectively). The supercapacitor retains a quasi-rectangular shaped CV curves at sweep rates lower than $50 \mathrm{mV} \mathrm{s}^{-1}$ but exhibit deviation at high scan rates indicating a high charge-transfer resistance (Fig. 4c). GCD curves were obtained at various current densities from 0.5 to $4 \mathrm{~A} \mathrm{~g}^{-1}$ to probe the cycling performance and capacitance retention. The charge-discharge curves were found to be symmetric triangles with no signs of significant self-discharge, indicating a highly reversible charge-discharge process (Fig. 4d). Specific capacitance $\left(C_{\mathrm{S}, \mathrm{GCD}}\right)$ of device showed a decrease from 603 to $55 \mathrm{~F} \mathrm{~g}^{-1}$ as the current density was (a)

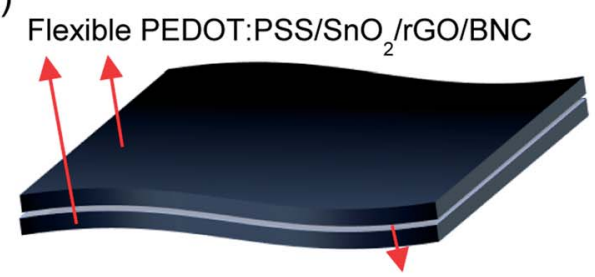

Gel Electrolyte coated BNC separator
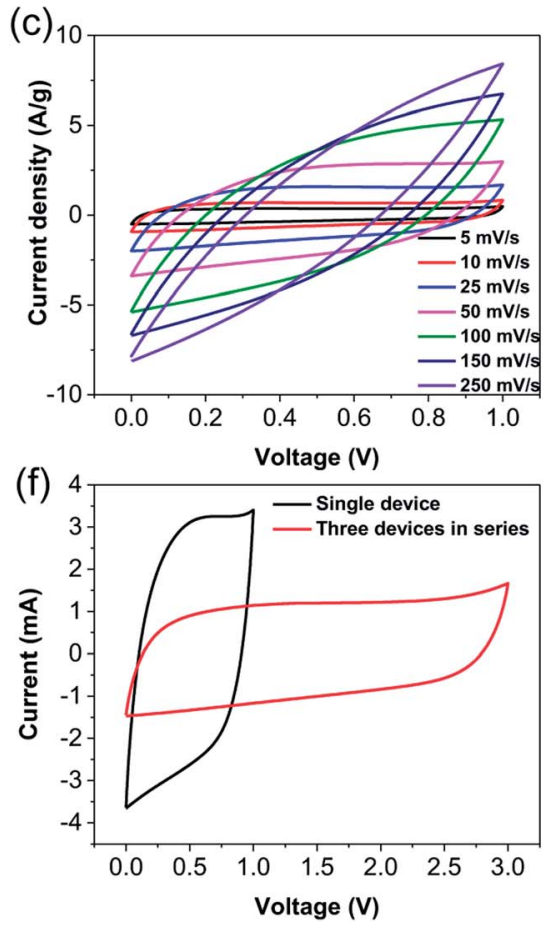

(b)

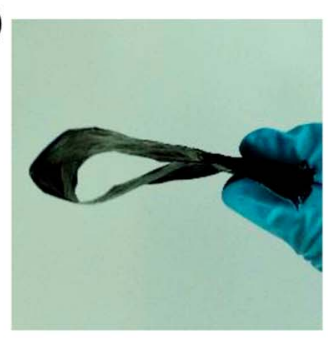

(d)
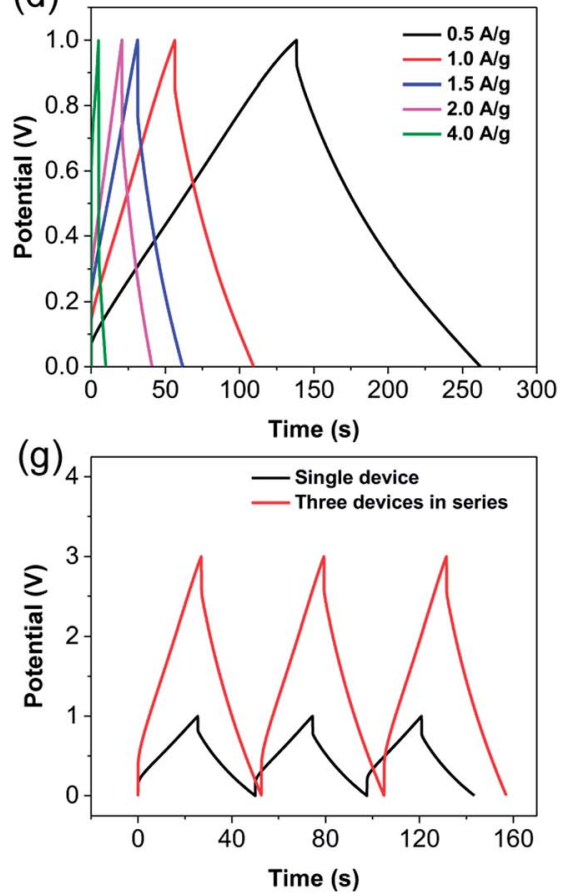
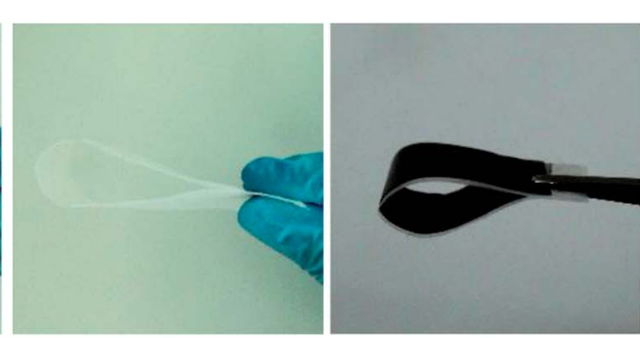

(e)

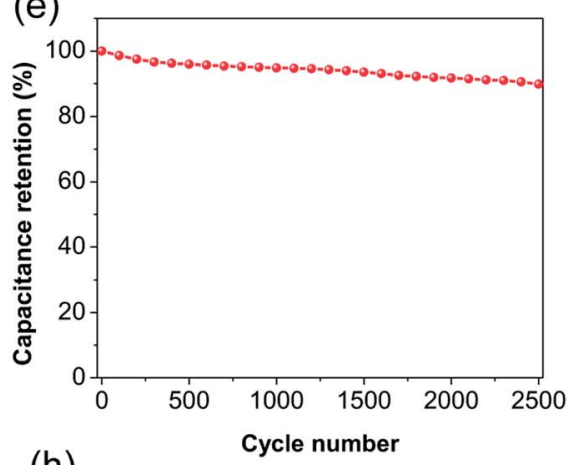

(h)

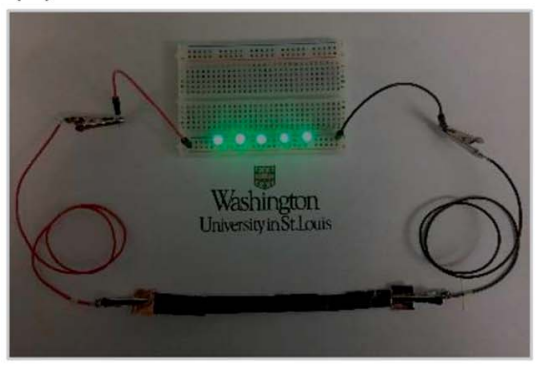

Fig. 4 (a) Schematic illustration of PEDOT:PSS/SnO $/ 2 / \mathrm{rGO} / \mathrm{BNC}$ electrodes based flexible solid-state supercapacitor. (b) Photograph of the electrode (left), BNC separator (middle), and flexible solid-state supercapacitor device (right). (c) CV curves of the supercapacitor at various scan rates. (d) Charge-discharge curves of the supercapacitor at various current densities. (e) Cycling performance of the PEDOT:PSS/SnO $/ \mathrm{rGO}$ BNC based supercapacitor. (f) CV curves of the supercapacitor at $50 \mathrm{mV} \mathrm{s}^{-1}$ and (g) charge-discharge curves at $2.0 \mathrm{~mA}$ of a single supercapacitor device (black) and three devices in series (red). (h) Photograph of five LEDs powered by the supercapacitors in series. 
increased from 0.5 to $4 \mathrm{~A} \mathrm{~g}^{-1}$ (Fig. S3†). CV curves of the supercapacitor device under different bending angles reveals the excellent flexibility and stability (Fig. S4†). Cycling performance was studied to determine the capacitance retention of the supercapacitor device over 2500 cycles at a charge rate of $2 \mathrm{~A} \mathrm{~g}^{-1}$. The device retained $89.8 \%$ capacitance after 2500 cycles demonstrating an excellent capacitance retention (Fig. 4e). Three devices were then connected in series to power a series of light emitting diodes. The CV curve of the three-device chain retains the rectangular shape even when charged to high voltage, indicating no large charge transfer resistances are added by connecting the devices in series (Fig. 4f). Similarly, GCD measurements of the three-devices in series show that the device chain can be charged in approximately the same amount of time as a single device, and retains the ideal symmetrical shape (Fig. 4g). Furthermore, the three-device chain of the flexible solid-state supercapacitor was charged and able to power a series of blue LEDs, demonstrating the potential application of this flexible energy storage device (Fig. 4h).

\section{Conclusion}

In conclusion, we have demonstrated a novel and facile method for the fabrication of PEDOT:PSS/ $\mathrm{SnO}_{2} / \mathrm{rGO} / \mathrm{BNC}$ electrodes for the fabrication of flexible, lightweight, and solid-state hybrid supercapacitor. The energy storage performance of the electrodes exhibited a significant improvement with the incorporation of $\mathrm{SnO}_{2}$ nanoparticles. The electrodes exhibit excellent electrochemical performance with specific capacitance of $445 \mathrm{~F} \mathrm{~g}^{-1}$ at the current density of $2 \mathrm{~A} \mathrm{~g}^{-1}$. The longevity and stability of PEDOT:PSS/SnO $/ 2 / \mathrm{rGO} / \mathrm{BNC}$ electrodes was evaluated by obtaining GCD curves and $84.1 \%$ of its original capacitance was retained after 2500 cycles at a current density of $2 \mathrm{~A} \mathrm{~g}^{-1}$. The materials and the fabrication process are scalable and cost-effective, which makes BNC-based supercapacitors promising candidates for flexible and wearable solid-state energy storage devices.

\section{Conflicts of interest}

There are no conflicts to declare.

\section{Acknowledgements}

This work was supported by Air Force Office of Scientific Research (AFOSR), award number: FA9550-15-1-0228. This work was performed in part at the Nano Research Facility (NRF), a member of the National Nanotechnology Infrastructure Network (NNIN). The authors thank Professor Julio M. D'Arcy in Department of Chemistry at Washington University for providing access to four-point probe station and thank Yang Lu for performing the experiment.

\section{Notes and references}

1 W. Liu, M.-S. Song, B. Kong and Y. Cui, Flexible and Stretchable Energy Storage: Recent Advances and Future Perspectives, Adv. Mater., 2017, 29, 1603436.
2 Z. Liu, Z.-S. Wu, S. Yang, R. Dong, X. Feng and K. Müllen, Ultraflexible In-Plane Micro-Supercapacitors by Direct Printing of Solution-Processable Electrochemically Exfoliated Graphene, Adv. Mater., 2016, 28, 2217-2222.

3 L. Wen, F. Li and H.-M. Cheng, Carbon Nanotubes and Graphene for Flexible Electrochemical Energy Storage: from Materials to Devices, Adv. Mater., 2016, 28, 4306-4337.

4 Q. Xue, J. Sun, Y. Huang, M. Zhu, Z. Pei, H. Li, Y. Wang, N. Li, H. Zhang and C. Zhi, Recent Progress on Flexible and Wearable Supercapacitors, Small, 2017, 1701827.

5 L. L. Zhang and X. S. Zhao, Carbon-based materials as supercapacitor electrodes, Chem. Soc. Rev., 2009, 38, 25202531.

6 G. Wang, L. Zhang and J. Zhang, A review of electrode materials for electrochemical supercapacitors, Chem. Soc. Rev., 2012, 41, 797-828.

7 L. Hu, J. W. Choi, Y. Yang, S. Jeong, F. La Mantia, L.-F. Cui and Y. Cui, Highly conductive paper for energy-storage devices, Proc. Natl. Acad. Sci., 2009, 106, 21490-21494.

8 K. Jost, C. R. Perez, J. K. McDonough, V. Presser, M. Heon, G. Dion and Y. Gogotsi, Carbon coated textiles for flexible energy storage, Energy Environ. Sci., 2011, 4, 5060-5067.

9 Y. Xu, Z. Lin, X. Huang, Y. Wang, Y. Huang and X. Duan, Functionalized Graphene Hydrogel-Based HighPerformance Supercapacitors, Adv. Mater., 2013, 25, 57795784.

10 H. Wang, B. Zhu, W. Jiang, Y. Yang, W. R. Leow, H. Wang and X. Chen, A Mechanically and Electrically Self-Healing Supercapacitor, Adv. Mater., 2014, 26, 3638-3643.

11 Q. Wang, J. Yan and Z. Fan, Carbon materials for high volumetric performance supercapacitors: design, progress, challenges and opportunities, Energy Environ. Sci., 2016, 9, 729-762.

12 M. Sawangphruk, P. Srimuk, P. Chiochan, A. Krittayavathananon, S. Luanwuthi and J. Limtrakul, High-performance supercapacitor of manganese oxide/ reduced graphene oxide nanocomposite coated on flexible carbon fiber paper, Carbon, 2013, 60, 109-116.

13 M. Sawangphruk, M. Suksomboon, K. Kongsupornsak, J. Khuntilo, P. Srimuk, Y. Sanguansak, P. Klunbud, P. Suktha and P. Chiochan, High-performance supercapacitors based on silver nanoparticle-polyanilinegraphene nanocomposites coated on flexible carbon fiber paper, J. Mater. Chem. A, 2013, 1, 9630-9636.

14 L. Yuan, X. Xiao, T. Ding, J. Zhong, X. Zhang, Y. Shen, B. Hu, Y. Huang, J. Zhou and Z. L. Wang, Paper-Based Supercapacitors for Self-Powered Nanosystems, Angew. Chem., Int. Ed., 2012, 51, 4934-4938.

15 C. Choi, H. J. Sim, G. M. Spinks, X. Lepró, R. H. Baughman and S. J. Kim, Elastomeric and Dynamic $\mathrm{MnO}_{2} / \mathrm{CNT}$ CoreShell Structure Coiled Yarn Supercapacitor, Adv. Energy Mater., 2016, 6, 1502119.

16 G. Yu, L. Hu, N. Liu, H. Wang, M. Vosgueritchian, Y. Yang, Y. Cui and Z. Bao, Enhancing the Supercapacitor Performance of Graphene/ $\mathrm{MnO}_{2} \quad$ Nanostructured Electrodes by Conductive Wrapping, Nano Lett., 2011, 11, 4438-4442. 
17 L. Huang, D. Chen, Y. Ding, S. Feng, Z. L. Wang and M. Liu, Nickel-Cobalt Hydroxide Nanosheets Coated on $\mathrm{NiCo}_{2} \mathrm{O}_{4}$ Nanowires Grown on Carbon Fiber Paper for HighPerformance Pseudocapacitors, Nano Lett., 2013, 13, 31353139.

18 X. Cao, Y. Shi, W. Shi, G. Lu, X. Huang, Q. Yan, Q. Zhang and H. Zhang, Preparation of Novel 3D Graphene Networks for Supercapacitor Applications, Small, 2011, 7, 3163-3168.

19 V. Augustyn, P. Simon and B. Dunn, Pseudocapacitive oxide materials for high-rate electrochemical energy storage, Energy Environ. Sci., 2014, 7, 1597-1614.

20 Y. Wang, Z. X. Huang, Y. Shi, J. I. Wong, M. Ding and H. Y. Yang, Designed hybrid nanostructure with catalytic effect: beyond the theoretical capacity of $\mathrm{SnO}_{2}$ anode material for lithium ion batteries, Sci. Rep., 2015, 5, 9164.

21 Y.-X. Wang, Y.-G. Lim, M.-S. Park, S.-L. Chou, J. H. Kim, H.-K. Liu, S.-X. Dou and Y.-J. Kim, Ultrafine $\mathrm{SnO}_{2}$ nanoparticle loading onto reduced graphene oxide as anodes for sodium-ion batteries with superior rate and cycling performances, J. Mater. Chem. A, 2014, 2, 529-534.

22 P. Simon and Y. Gogotsi, Materials for electrochemical capacitors, Nat. Mater., 2008, 7, 845-854.

23 J. R. Miller and P. Simon, Electrochemical Capacitors for Energy Management, Science, 2008, 321, 651-652.

24 Y. Ko, M. Kwon, W. K. Bae, B. Lee, S. W. Lee and J. Cho, Flexible supercapacitor electrodes based on real metal-like cellulose papers, Nat. Commun., 2017, 8, 536.

25 Z. Wang, P. Tammela, M. Strømme and L. Nyholm, Cellulose-based Supercapacitors: Material and Performance Considerations, Adv. Energy Mater., 2017, 7, 1700130.

26 Z. Gui, H. Zhu, E. Gillette, X. Han, G. W. Rubloff, L. Hu and S. B. Lee, Natural Cellulose Fiber as Substrate for Supercapacitor, ACS Nano, 2013, 7, 6037-6046.

27 L. Hu and Y. Cui, Energy and environmental nanotechnology in conductive paper and textiles, Energy Environ. Sci., 2012, 5, 6423-6435.

28 L. Yuan, B. Yao, B. Hu, K. Huo, W. Chen and J. Zhou, Polypyrrole-coated paper for flexible solid-state energy storage, Energy Environ. Sci., 2013, 6, 470-476.

29 L. Liu, Z. Niu, L. Zhang, W. Zhou, X. Chen and S. Xie, Nanostructured Graphene Composite Papers for Highly
Flexible and Foldable Supercapacitors, Adv. Mater., 2014, 26, 4855-4862.

30 A. Razaq, L. Nyholm, M. Sjödin, M. Strømme and A. Mihranyan, Paper-Based Energy-Storage Devices Comprising Carbon Fiber-Reinforced PolypyrroleCladophora Nanocellulose Composite Electrodes, Adv. Energy Mater., 2012, 2, 445-454.

31 C. Chen, Y. Zhang, Y. Li, J. Dai, J. Song, Y. Yao, Y. Gong, I. Kierzewski, J. Xie and L. Hu, All-wood, low tortuosity, aqueous, biodegradable supercapacitors with ultra-high capacitance, Energy Environ. Sci., 2017, 10, 538-545.

32 Q. Jiang, C. Kacica, T. Soundappan, K.-k. Liu, S. Tadepalli, P. Biswas and S. Singamaneni, An in situ grown bacterial nanocellulose/graphene oxide composite for flexible supercapacitors, J. Mater. Chem. A, 2017, 5, 13976-13982.

33 D. C. Marcano, D. V. Kosynkin, J. M. Berlin, A. Sinitskii, Z. Sun, A. Slesarev, L. B. Alemany, W. Lu and J. M. Tour, Improved Synthesis of Graphene Oxide, ACS Nano, 2010, 4, 4806-4814.

34 H. D. Pham, V. H. Pham, T. V. Cuong, T.-D. Nguyen-Phan, J. S. Chung, E. W. Shin and S. Kim, Synthesis of the chemically converted graphene xerogel with superior electrical conductivity, Chem. Commun., 2011, 47, 96729674.

35 Y. Lu, L. M. Santino, S. Acharya, H. Anandarajah and J. M. D'Arcy, Studying Electrical Conductivity Using a 3D Printed Four-Point Probe Station, J. Chem. Educ., 2017, 94, 950-955.

36 C. Zhang, H. B. Wu, C. Yuan, Z. Guo and X. W. Lou, Confining Sulfur in Double-Shelled Hollow Carbon Spheres for Lithium-Sulfur Batteries, Angew. Chem., Int. Ed., 2012, 51, 9592-9595.

37 R. Tian, Y. Zhang, Z. Chen, H. Duan, B. Xu, Y. Guo, H. Kang, $\mathrm{H}$. $\mathrm{Li}$ and $\mathrm{H}$. Liu, The effect of annealing on a $3 \mathrm{D} \mathrm{SnO}_{2} /$ graphene foam as an advanced lithium-ion battery anode, Sci. Rep., 2016, 6, 19195.

38 W. Wang, Q. Hao, W. Lei, X. Xia and X. Wang, Graphene/ $\mathrm{SnO}_{2}$ /polypyrrole ternary nanocomposites as supercapacitor electrode materials, RSC Adv., 2012, 2, 10268-10274. 